\title{
Mass and metallicity distribution of parent AGB stars of presolar SiC
}

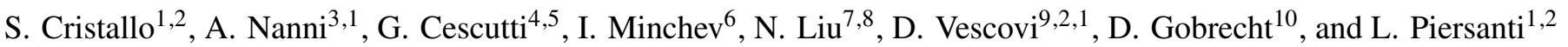

1 INAF, Osservatorio Astronomico d'Abruzzo, Via Mentore Maggini snc, 64100 Teramo, Italy

e-mail: sergio.cristallo@inaf.it

2 INFN, Sezione di Perugia, Via A. Pascoli snc, 06123 Perugia, Italy

3 Aix Marseille Univ., CNRS, CNES, LAM, Marseille, France

${ }^{4}$ INAF, Osservatorio Astronomico di Trieste, Via G.B. Tiepolo 11, 34143 Trieste, Italy

5 IFPU, Istitute for the Fundamental Physics of the Universe, Via Beirut 2, 34151 Grignano, Trieste, Italy

${ }^{6}$ Leibniz Institut für Astrophysik Potsdam (AIP), An der Sterwarte 16, 14482 Potsdam, Germany

7 Laboratory for Space Sciences and Physics Department, Washington University in St. Louis, St. Louis, MO 63130, USA

8 McDonnell Center for the Space Sciences, Washington University in St. Louis, St. Louis, MO 63130, USA

9 Gran Sasso Science Institute, Viale Francesco Crispi, 7, 67100 L'Aquila, Italy

${ }^{10}$ Institute of Astronomy, KU Leuven, Celestijnenlaan 200D, 3001 Leuven, Belgium

Received 22 September 2020 / Accepted 16 October 2020

\section{ABSTRACT}

\begin{abstract}
The vast majority ( $\gtrsim 90 \%$ ) of presolar SiC grains identified in primitive meteorites are relics of ancient asymptotic giant branch (AGB) stars, whose ejecta were incorporated into the Solar System during its formation. Detailed characterization of these ancient stardust grains has revealed valuable information on mixing processes in AGB interiors in great detail. However, the mass and metallicity distribution of their parent stars still remains ambiguous, although such information is crucial to investigating the slow neutroncapture process, whose efficiency depends on mass and metallicity. Using a well-known Milky Way chemo-dynamical model, we followed the evolution of the AGB stars that polluted the Solar System at $4.57 \mathrm{Gyr}$ ago and weighted the stars based on their SiC dust productions. We find that presolar $\mathrm{SiC}$ in the Solar System predominantly originated from AGB stars with $M \sim 2 M_{\odot}$ and $Z \sim Z_{\odot}$. Our finding well explains the grain-size distribution of presolar SiC identified in situ in primitive meteorites. Moreover, it provides complementary results to very recent papers that characterized parent stars of presolar SiC.
\end{abstract}

Key words. stars: AGB and post-AGB - circumstellar matter

\section{Introduction}

Stars are very efficient nuclear cauldrons, since they synthesize the vast majority of chemical elements in the Universe. Isotopes that are freshly synthesized in their interiors are directly ejected (in case of explosive events) into the interstellar medium (ISM) or are carried to the surface by convective mixing episodes (known as dredge-ups) and are then lost to the ISM by stellar winds. In stars, elements heavier than iron are mostly produced in two neutron-capture processes: the rapid process ( $r$-process; see Cowan et al. 2019 for a review), and the slow process ( $s$-process; see Busso et al. 1999 for a review). The latter mainly occurs during the asymptotic giant branch (AGB) phase of lowmass stars (see, e.g., Straniero et al. 2006).

Isotopic ratios of $s$-process elements can be obtained in single $\mu \mathrm{m}$-sized presolar $\mathrm{SiC}$ grains with very high precisions $(<10 \%$ errors; Savina et al. 2003; Stephan et al. 2016). The majority $(\gtrsim 90 \%)$ of presolar $\mathrm{SiC}$ grains identified in extraterrestrial materials, including mainstream, $Y$, and $Z$ grains (AGB grains hereafter), came from ancient $C$-rich AGB stars that evolved prior to the formation of the Solar System (Zinner et al. 2014). Compared to mainstream grains, $Y$ and $Z$ grains are much rarer (5\% of all $\mathrm{SiC}$ each) and their abundances increase with decreasing grain size (Zinner et al. 2014; Hoppe et al. 2010; $\mathrm{Xu}$ et al. 2015). Based on their $\mathrm{Si}$ and $\mathrm{Ti}$ isotopic compositions, $Y$ and $Z$ grains have previously been inferred to have origi- nated in low-mass AGB stars of $0.5 Z_{\odot}$ and $0.3 Z_{\odot}$, respectively (Zinner et al. 2007). However, Liu et al. (2019) showed that the low-metallicity models adopted by Zinner et al. (2007) cannot consistently explain the Mo isotopic compositions of $Y$ and $Z$ grains and that, more likely, $Y$ and $Z$ grains came from low-mass AGB stars of $\gtrsim 0.7 Z_{\odot}$. A small fraction of presolar $\mathrm{SiC}$, including $X$ and possibly AB grains, also came from ancient type II supernovae (Zinner et al. 2014; Liu et al. 2017a; Hoppe et al. 2019). These stellar dust grains were incorporated into small bodies that formed shortly after the Sun's birth. Billions of years later, primitive extraterrestrial materials, fragments of undifferentiated small bodies (e.g., asteroids, and to a lesser extent, comets), fell and delivered their initially incorporated ancient stellar dust to Earth. The linkage between these AGB grains and C-rich AGB stars is particularly supported by the $s$-process isotopic signatures preserved in the grains, which have provided a number of stringent constraints on the nucleosynthesis occurring in AGB interiors (e.g., Lugaro et al. 2003; Liu et al. 2014, 2015, 2019).

Unfortunately, the distribution (i.e., masses and metallicities) of the parent stars of AGB grains remains unknown because we lack direct observations, and it thus needs to be inferred based on Galactic evolution models. Gail et al. (2009) presented a Galactic chemical evolution (GCE) model with dust yields computed with synthetic AGB models, based on which they concluded that the majority of presolar $\mathrm{SiC}$ grains originated in AGB stars with masses $1.5 \leq M / M_{\odot} \leq 4.0$ and roughly solar 


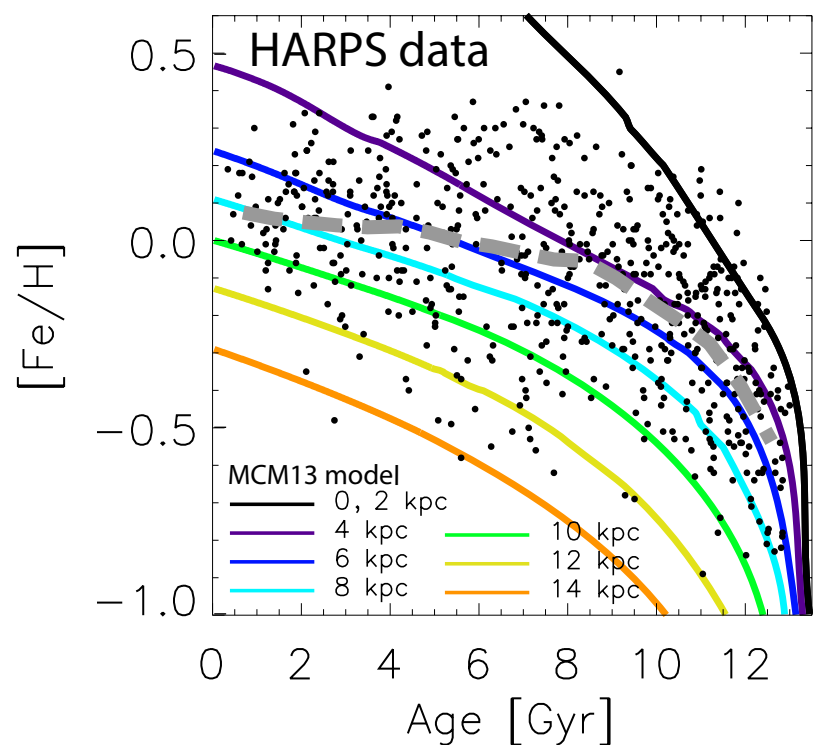

Fig. 1. Scatter points are HARPS CTO data (recently reanalyzed data by Delgado Mena et al. 2017 and age estimates by Anders et al. 2018), which represent the AMR in the Galaxy. The dashed gray line shows the average of the total sample, which appears flat in the last $\sim 5 \mathrm{Gyr}$. The color-coded curves indicate averages of subsamples according to their birth locations from the MCM13 model. The apparent flatness in the local AMR can be explained by the superposition of well-defined AMRs of stars born in narrow birth-radius bins.

metallicity. More recent studies focusing on the isotopic compositions of AGB grains suggested that AGB grains predominantly came from low-mass stars $\left(\lesssim 3 M_{\odot}\right.$ ) with close-to-solar metallicities (e.g., Liu et al. 2018, 2019) or slightly supersolar metallicity (Lewis et al. 2013). Finally, Lugaro et al. (2018, 2020) proposed AGB stars with higher masses $\left(M / M_{\odot} \sim 3.5-4\right)$ and higher metallicities $\left(Z / Z_{\odot} \sim 1.5-2\right)$ as the parent stars of large $(>1 \mu \mathrm{m}$ in diameter) mainstream grains.

We aim to constrain the mass and metallicity distribution of the parent stars of the grains by coupling chemo-dynamical, stellar, and dust growth codes. This will help to distinguish among the proposed $s$-process models in the literature.

\section{Milky Way chemo-dynamical model}

To quantify the number of AGB stars close to the Sun's birth place at the epoch of its formation, we used the chemodynamical model by Minchev et al. (2013; hereafter MCM13). This model was created by combining a high-resolution simulation in the cosmological context with a detailed Milky Way chemical evolution model. The simulation used here was part of a suite of numerical experiments presented by Martig et al. (2012), in which the authors studied the evolution of 33 simulated galaxies from redshift $z=5$ to $z=0$. To avoid problems with subgrid physics in fully cosmological chemo-dynamical models (i.e., with on-the-fly star formation and chemical enrichment), a detailed semianalytical chemical evolution model for the Milky Way was coupled with the simulation a posteriori, assigning elemental abundances to stars according to their ages, birth radii, and the weighted star formation history. The unconstrained nature of the simulation allows stars to migrate radially in a fully self-consistent matter. Because it is impossible to follow single stars during their migration, they are grouped in "star particles" (hereafter SPs), each with a mass of $7.5 \times 10^{4} M_{\odot}$. The corresponding hybrid chemo-dynamical model succeeded

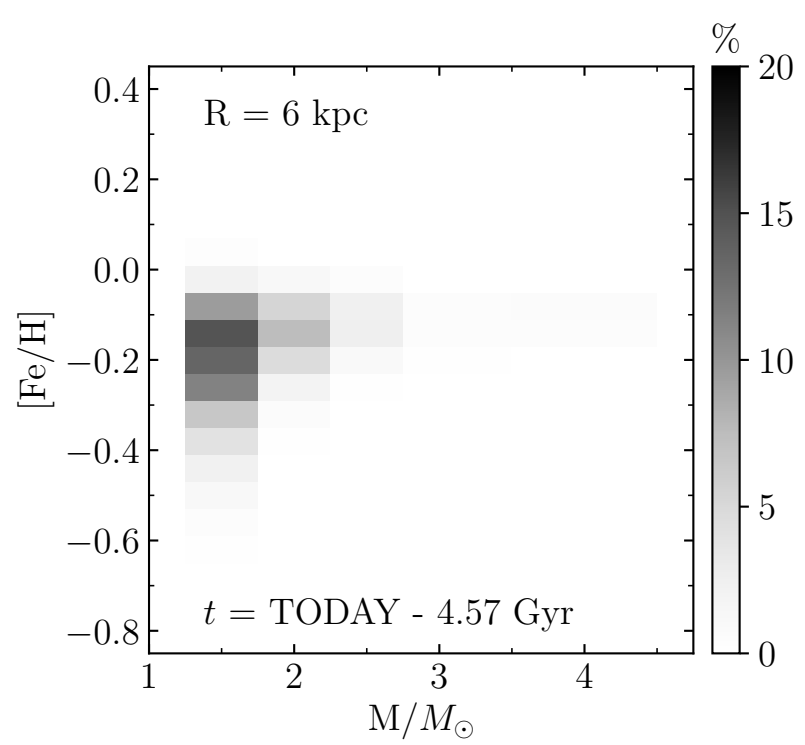

Fig. 2. Contour plot of the stellar mass-metallicity distribution in the solar vicinity at the epoch of Solar System formation (MCM13 model output).

in reproducing the chemo-kinematic relations in the Milky Way (see Minchev 2016 and references therein).

We expect that stars attaining their AGB phase in the solar vicinity at the epoch of Sun formation $(4.57 \mathrm{Gyr}$ ago $)$ produced the vast majority of presolar $\mathrm{SiC}$ grains. It is possible that some dust that was produced in AGB stars evolved far from the Sun's birth place, arrived in the solar vicinity with different delays (on average fewer than $300 \mathrm{Myr}$ prior to the Sun's birth; Heck et al. 2020). However, the number of such migrated grains is thought to be very small because the isotropic emission at the source (matter ejected by low-mass stars has no preferential direction) caused strong dilution and because the most energetic radiation permeating the ISM (probably) destroyed them.

The almost flat age-metallicity relation (AMR) in the solar neighborhood for the last 5 Gyr (dashed gray curve in Fig. 1) seems to suggest that the metallicity distribution of the Galactic disk barely evolved over time ${ }^{1}$. However, the temporal evolution of the metallicities of the local stars is also driven by dynamical processes such as stellar migration, which modifies the distribution of stars in the solar vicinity over time. The local AMR is largely controlled by long-lived stars, a large fraction of which migrate out from the inner disk, where the metallicity for a given age is higher. This effect is illustrated in Fig. 1: the apparent flatness of the observed AMR in the solar vicinity (HARPS-GTO data) can be explained by the MCM13 model as the result of radial migration and the superposition of stars born at different Galactic radii. As indicated by the color-coded curves in Fig. 1, stars born in narrow birth-radius bins have well-defined AMRs. However, when put together, the total sample appears much flatter. This is a case of the statistical phenomenon known as Simpson's paradox (see Minchev et al. 2019).

The mass-metallicity probability distribution of AGB stars present in the solar neighborhood at the epoch of its formation (4.57 Gyr ago) obtained with the MCM13 model is shown in Fig. 2. We assumed that the Sun formed at an inner Galactocentric radius $(6 \mathrm{Kpc})$ and subsequently migrated to its current radius of $8 \mathrm{kpc}$ (Wielen et al. 1996; Clayton 1997; MCM13).

1 On the $x$-axis, time $t=0$ yr refers to the current date. 
The grid resolution is determined by the resolutions of the masses and metallicities of the available AGB stellar models contained in the FRUITY database ${ }^{2}$ (Cristallo et al. 2009, 2011, 2015; Piersanti et al. 2013). In the framework of our AGB models, we did not consider stars with masses below $1.3 M_{\odot}$ because they do not experience enough third dredge-up (TDU) to become C-rich (except for very low metallicities, which are irrelevant for this study). The MCM13 model was used to directly output the metallicity distribution of considered SPs in the solar vicinity at 4.57 Gyr ago, as shown in Fig. 2.

The determination of the corresponding mass distribution was more complex. In order to obtain the mass distibution, we assumed that each SP consisted of a simple stellar population. The distribution of SPs in the solar vicinity at the epoch of the Sun formation is already given by the MCM13 model, but we also need to know the number of objects on the AGB phase within each of the SPs, which is intrinsically connected to the SP age. Because this is a simple stellar population, the mass evolving on the AGB is univocally determined for each SP. For example, only SPs older than 10 Gyr can host AGB stars with initial masses below $1 M_{\odot}$. In order to extract this quantity, we grouped SPs in temporal bins, which were defined based on the timescale needed for a star to reach the AGB (depending on the initial stellar mass). For instance, a $1.5 M_{\odot}$ star needs approximately 3 Gyr to attain the AGB phase, while a $6.0 M_{\odot}$ only needs about 70 Myr. We know the distribution of SPs in each temporal bin, therefore in order to extract the number of AGB stars from each SP, we applied a Salpeter initial mass function (IMF; Salpeter 1955).

The predicted distribution in Fig. 2 is largely weighted toward $1.5 M_{\odot}$ mass at lower-than-solar metallicities $(Z \simeq$ $\left.0.7 Z_{\odot}\right)^{3}$. This is a direct consequence of the IMF peaking at extremely low masses. Finally, to investigate their contributions to the presolar SiC inventory in the Solar System, these AGB stars in Fig. 2 have to be further weighted by their SiC dust production.

\section{Dust growth model}

In order to determine SiC yields in AGB stars, we adopted a dust growth code, coupled with wind dynamics, initially based on Ferrarotti \& Gail (2006), but with some important modifications in the input physics (Nanni et al. 2013, 2016; Nanni 2019). For each mass-metallicity combination identified by the MCM13 model, we calculated the corresponding dust yield. The reference stellar evolutionary models were taken from the FRUITY database. For each model we extracted physical inputs along the AGB track (i.e., luminosity, current stellar mass, temperature, mass-loss rate, and surface chemical abundances). As output, we obtain the chemical composition of the produced dust, the relative yields of different dust species, and the expansion velocity of the external layers of the circumstellar envelope.

We introduced a time-averaged shock density profile in the inner part of the envelope before the onset of the dust-driven wind, starting from the prescriptions by Cherchneff et al. (1992). This modification is necessary to reliably model the condensation of $\mathrm{SiC}$, which is condensed at higher temperatures than carbon (amorphous) dust. The formation of this dust species, under favorable conditions, accelerates the outflow through a dust-driven wind. In particular, the original stationary wind

\footnotetext{
http://fruity.oa-abruzzo.inaf.it/

In FRUITY models, $Z_{\odot}=1.4 \times 10^{-2}$.
}

profile underestimates the density at the formation radius of $\mathrm{SiC}$, making it difficult to produce large $\mathrm{SiC}$ grains (highlighted by laboratory measurements; see the next section).

In order to describe the circumstellar envelope, we operatively ideally divided it into three spatially separated regions: a) from the photosphere to the condensation radius, $R_{\text {cond }}$, where the first dust species form; b) from $R_{\text {cond }}$ to the radius at which the outflow starts to accelerate through dust-driven wind, $R_{\mathrm{acc}}$; and c) from $R_{\mathrm{acc}}$ outward. For the temperature profile from $R_{\text {cond }}$ outward, we adopted the Lucy approximation (Lucy 1976), which has been used in several other works (e.g., Ferrarotti \& Gail 2006; Ventura et al. 2012; Nanni et al. 2013). To describe the inner temperature profile from the photosphere to $R_{\text {cond }}$, we adopted the following temperature profile:

$T_{\text {inner }}(r)=T_{\text {eff }}\left(\frac{r}{R_{*}}\right)^{-\alpha_{\mathrm{T}}}$,

where $T_{\text {eff }}$ is the effective temperature, $R_{*}$ the stellar radius and $\alpha_{\mathrm{T}}$ is a parameter determined by linking the inner and outer temperature profiles at $R_{\text {cond }}$.

To describe the density profile in the region with dust-driven wind in region c), the stationary wind equation was adopted (e.g., Ferrarotti \& Gail 2006; Ventura et al. 2012; Nanni et al. 2013). To describe the density profile in regions a) and b), we adopted the time-averaged approximation for the shock-extended zone presented in Cherchneff et al. (1992),

$\rho(r)=\rho_{0} \times \exp \int_{R^{*}}^{r}-\frac{\left(1-\gamma_{\text {shock }}^{2}\right)}{H_{0}\left(r^{\prime}\right)} \mathrm{d} r^{\prime}$,

where $R_{*}$ is the photospheric radius, $\rho_{0}$ is the value of the density at the photosphere, and $\gamma_{\text {shock }}$ is the shock strength and is evaluated by linking the inner density profile with the density value obtained at $R_{\text {acc }}$ from the stationary wind profile. The quantity $H_{0}(r)$ is given by

$H_{0}(r)=\frac{k T(r) r^{2}}{\mu m_{\mathrm{H}} G M_{*}}$,

where $k$ is the Boltzmann constant, $T(r)$ the temperature profile, $\mu$ is the mean molecular weight, $m_{\mathrm{H}}$ is the mass of hydrogen atom, $G$ is the gravity constant, and $M_{*}$ is the stellar mass. The density profile was numerically evaluated by performing the integral of $H_{0}(r)$ in Eq. (2). We note that we assumed a different temperature profile with respect to Cherchneff et al. (1992), who chose a fixed value for the parameter $\alpha=0.6$ (assumed representative for describing the temperature profile of the circumstellar envelope of the carbon star IRC+10216). Because we needed to simulate a variety of carbon stars that evolved through the AGB phase, the adoption of the same type of temperature profile is not straightforward: we would need to predict how $\alpha$ changes as a function of the stellar parameters. Therefore we adopted the temperature profile by Lucy (1976) from $R_{\text {cond }}$ outward, which was consistently computed taking the amount of dust that formed in the circumstellar envelope as a function of the stellar parameters into account. In order to compute the integral of $H_{0}(r)$ in Eq. (2), we then adopted the temperature profile from the photosphere to $R_{\text {cond }}$ given by Eq. (1), consistently matched with the outer temperature profile.

We modeled the grain growth process on starting seed nuclei of two types of carbonaceous grains: amorphous carbon, and SiC. For amorphous carbon, the seed particle abundance $\epsilon_{\mathrm{C}}$, 

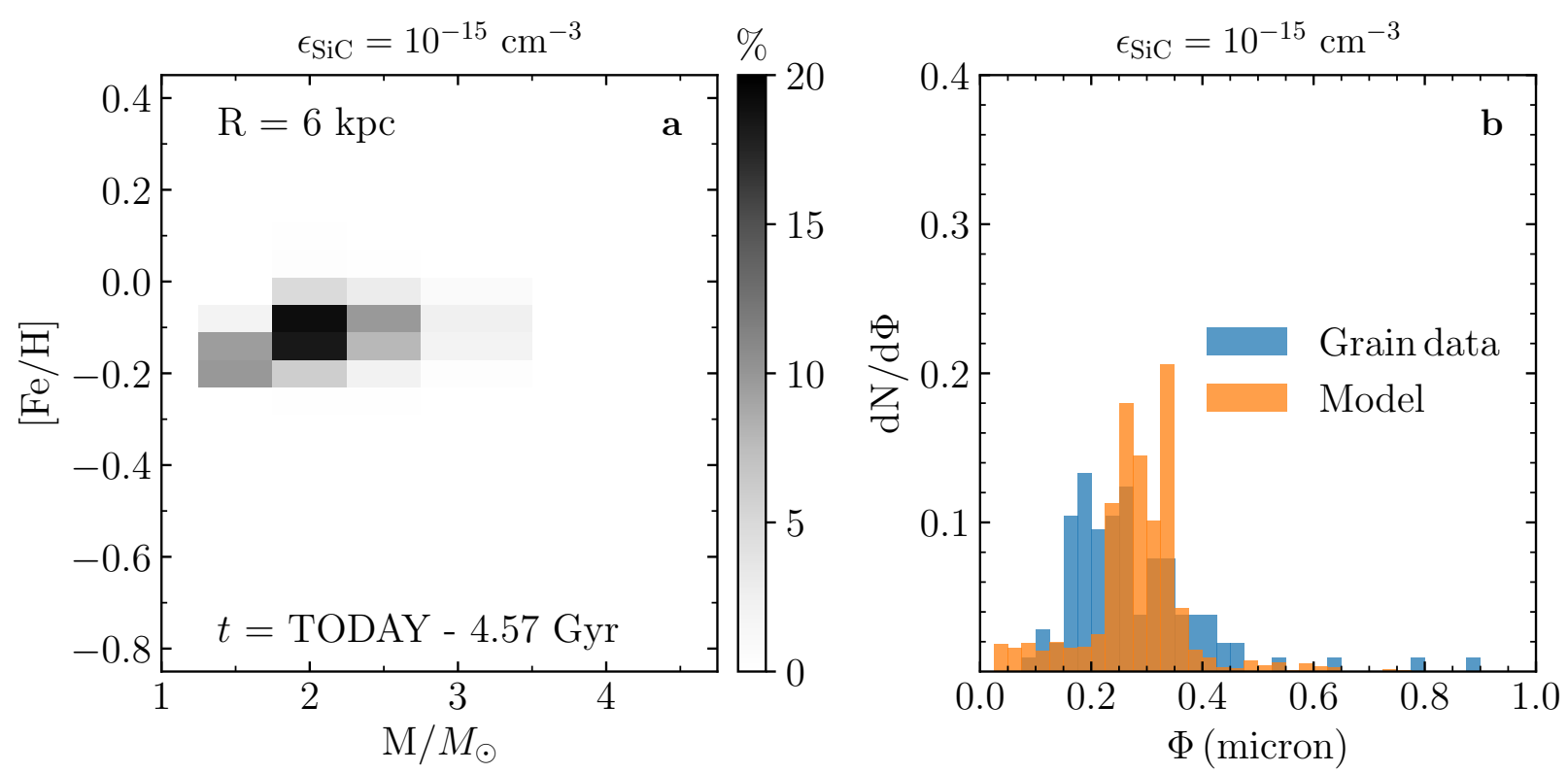

Fig. 3. Panel a: same as Fig. 2, but the percentages are weighted by the $\mathrm{SiC}$ grain yields of the corresponding AGB models (initial seed dust number $\epsilon_{\mathrm{SiC}}=10^{-15} \mathrm{~cm}^{-3}$ ). Panel $b$ : corresponding distribution of $\mathrm{SiC}$ production vs. grain size (in diameter), compared to SiC grains identified in situ in primitive meteorites (see text for details).

that is, the abundance of particles with respect to hydrogen atoms on which other molecules accrete, was selected in order to yield typical dust grains of size $\lesssim 0.04 \mu \mathrm{m}$. The value of $\epsilon_{\mathrm{C}}$ is proportional to the carbon excess: $\epsilon_{\mathrm{C}}=\epsilon_{\mathrm{C}, 0} \times(\mathrm{C}-\mathrm{O})$. The values of $\epsilon_{\mathrm{C}, 0}$ together with optical constants from Hanner (1988) were selected to reproduce both the photometry in the near- and mid-infrared bands (Nanni et al. 2016) and Gaia Data Release 2 (Nanni 2019). The condensation of amorphous carbon was assumed to occur by a successive addition of $\mathrm{C}_{2} \mathrm{H}_{2}$ molecules to the seed particle below a temperature of 1100 K (Frenklach \& Feigelson 1989; Cherchneff et al. 1992). The probability for molecules (in our case $\mathrm{C}_{2} \mathrm{H}_{2}$ ) to stick on the grain surface is known as "sticking coefficient". These coefficients cannot be derived from theoretical calculations and are poorly constrained by laboratory experiments. We initially adopted a sticking coefficient equal to unity for amorphous carbon (as well as for $\mathrm{SiC}$, see below). However, with this choice, we were unable to reproduce the observed expansion velocity versus mass-loss rates observed for Galactic carbon stars. As already highlighted, carbon dust is responsible for the outflow acceleration: a sticking coefficient equal to unity would lead to an outflow expansion velocity higher than the observed ones. On the other hand, a sticking coefficient of 0.2 reduces the expansion velocity by lowering the amount of amorphous carbon, and coupled to an initial wind speed of $4 \mathrm{~km} \mathrm{~s}^{-1}$, produces final velocity profiles that are consistent with observations.

$\mathrm{SiC}$ formation is assumed to proceed through the addition of $\mathrm{Si}$ atoms and $\mathrm{C}_{2} \mathrm{H}_{2}$ molecules on the seed. We maintained the original value of a sticking coefficient for $\mathrm{SiC}$ equal to unity. A variation in the sticking coefficient for $\mathrm{SiC}$ does not affect the outflow expansion velocity because the formation of $\mathrm{SiC}$ is not responsible for accelerating the outflow. We did not find valid reasons to modify the $\mathrm{SiC}$ sticking coefficient, especially considering the lack of observational constraints. The condensation of $\mathrm{SiC}$ is prevented at high temperatures by chemisputtering, which is the destructive bombardment of a solid by molecular hydrogen $\left(\mathrm{H}_{2}\right)$. Below a certain temperature, which is model dependent, destruction becomes inefficient and $\mathrm{SiC}$ grains can thus grow unhindered. We adopted the optical constants from Pitman et al. (2008) for $\beta$-SiC because structural analyses of presolar $\mathrm{SiC}$ grains revealed that they consist dominantly of $\beta$-SiC, also known as cubic SiC (3C-SiC; Daulton et al. 2002; Liu et al. 2017b). For the seed particle abundance, we chose $\epsilon_{\mathrm{SiC}}=10^{-15}$. In order to evaluate the sensitivity of our results to this free parameter, we also tested significantly higher $\left(\epsilon_{\mathrm{SiC}}=\right.$ $\left.10^{-13}\right)$ and lower $\left(\epsilon_{\mathrm{SiC}}=10^{-18}\right)$ values (see the next section). We expect some degeneracy between the results obtained with a large sticking coefficient and a low seed particle abundance and the results obtained with a low sticking coefficient and a large seed particle abundances. For this reason, we varied one parameter alone $\left(\epsilon_{\mathrm{SiC}}\right)$, which in any case produces the largest differences as far as the total predicted mass of $\mathrm{SiC}$ dust is concerned.

\section{Results and discussions}

When the SiC dust yields were calculated based on FRUITY models, these values were used to assign weights to the percentages obtained with the chemo-dynamical MCM13 model (Fig. 2). The weighted SiC production distribution is shown in Fig. $3 \mathrm{a}$, which reveals a number of interesting features as summarized below:

1. Because this study focuses on SiC dust, only C-rich models were considered in our simulations. This led to a reduced number of considered mass and metallicity combinations. As a result, the $1.5 M_{\odot}$ stars at high metallicities, and in general, stars more massive than $3 M_{\odot}$, were thus excluded from our simulations because they are predicted to be oxygen-rich by FRUITY stellar models. Although this prediction depends strictly on the TDU efficiency of our stellar models, the number of these stellar objects is negligible in the solar neighborhood at $4.57 \mathrm{Gyr}$ ago according to the MCM13 modeling result. In other words, our results shown in Fig. 3 are barely affected by these stellar objects even if they could become carbon-rich.

2. Figure 3 a illustrates that $\sim 2 M_{\odot}$ AGB stars are the dominant contributors to AGB grains in the Solar System, resulting 
from the fact that they were numerous at the epoch of its formation and are predicted to dredge-up more material than lower mass stars (see, e.g., Cristallo et al. 2011).

3. Figure 3 a reveals a general shift to higher metallicities than in Fig. 2. This can be easily understood in terms of the $\mathrm{SiC}$ formation process, which is governed by carbon and silicon abundances. While the former depends on the TDU efficiency, the latter is directly proportional to the metallicity of the model. Thus, the higher the metallicity, the higher the expected $\mathrm{SiC}$ yield. It is noteworthy that an additional production of ${ }^{12} \mathrm{C}$, which occurs at lower metallicities, does not directly translate into a higher production of $\mathrm{SiC}$ because the carbon excess tends to form additional amorphous carbon (see Nanni et al. 2013 and references therein).

By extracting presolar SiC grains from Murchison meteorite through acid dissolution, Amari et al. (1994) studied the grain size distribution and found that the grain size distribution has a maximum at $0.4 \mu \mathrm{m}$, and follows a power law with an exponent between -4 and -5 for sizes between 0.7 and $3.2 \mu \mathrm{m}$. This estimate, however, is likely skewed toward larger sizes because the extraction of smaller grains is expected to be less efficient. In addition to the acid-extraction method, presolar SiC grains can also be directly identified in situ in primitive meteorites by isotopic mapping. Such samples should better represent the whole population of AGB grains incorporated during the Solar System formation. We calculated the size distribution of AGB grains identified in situ in the most primitive meteorites (Floss \& Stadermann 2009; Nguyen et al. 2010; Nittler et al. 2018; Haenecour et al. 2018) to eliminate the effect of parentbody processing on varying the grain size (Davidson et al. 2014). The result (blue histogram) is compared to our model predictions (orange histogram), in Fig. 3b. The model result overlaps the grain data with a small shift to larger grains (by $\sim 100 \mathrm{~nm}$ ).

In order to strengthen our results, we tested higher and lower initial seed particle abundances $\left(\epsilon_{\mathrm{SiC}}=10^{-13}\right.$ and $10^{-18} \mathrm{~cm}^{-3}$, in comparison to $\epsilon_{\mathrm{SiC}}=10^{-15} \mathrm{~cm}^{-3}$ in Fig. 4) in our calculations. The results are shown in Figs. $4 \mathrm{~b}$ and $\mathrm{d}$. The $\epsilon_{\mathrm{SiC}}=10^{-13} \mathrm{~cm}^{-3}$ case matches the small-size tail of the grain distribution. On the other hand, the $\epsilon_{\mathrm{SiC}}=10^{-18} \mathrm{~cm}^{-3}$ case predicts the formation of large grains, a consistent fraction of which ranges from 0.5 to $\sim 1 \mu \mathrm{m}$ in size (thus larger than grain data). Thus, $\epsilon_{\mathrm{SiC}}=10^{-18} \mathrm{~cm}^{-3}$ can be considered as a lower limit for the seed particle abundance. The corresponding mass-metallicity distributions of the AGB stars are reported in Figs. 4a and c. Based on Figs. 3 and 4, we conclude that $\mathrm{AGB}$ stars with $M \sim 2 M_{\odot}$ and $Z \sim Z_{\odot}$ are the dominant contributors to AGB grains identified in primitive extraterrestrial materials. The predicted distributions of AGB stars in Figs. 3a, 4a, and 4c also provide a natural explanation to the abundances and isotopic compositions of $Y$ and $Z$ grains. $Y$ and $Z$ grains were constrained to have come from low-mass AGB stars of $\gtrsim 0.7 Z_{\odot}$ based on comparison of their Mo isotopic compositions with FRUITY model predictions (Liu et al. 2019). Both the lower limit of their parent star metallicities and their low abundances are consistent with the decreasing probabilities of low-mass AGB stars toward lower metallicities $\left([\mathrm{Fe} / \mathrm{H}]=-0.2\right.$, corresponding to $0.63 Z_{\odot}$, represents a sort of lower limit of our metallicity distribution). The metallicity distribution only varies slightly from $\epsilon_{\mathrm{SiC}}=10^{-13} \mathrm{~cm}^{-3}$ to $\epsilon_{\mathrm{SiC}}=10^{-18} \mathrm{~cm}^{-3}$, thus demonstrating that our conclusions are barely affected by the choice of $\epsilon_{\mathrm{SiC}}$ (which is by far the largest uncertainty source of the $\mathrm{SiC}$ dust growth process). We demonstrated that the distribution of parent AGB stars of presolar grain is dominantly shaped by the result of the MCM13 model.
Except for the differences in the physical recipes of the dust growth models (see the discussion in Sect. 3), our conclusions are similar to the results by Gail et al. (2009). Nonetheless, some differences have to be highlighted. In particular, in the study of Gail et al. (2009), the parent AGB star distribution is characterized by lower metallicities and higher masses. First, we note that a standard GCE model (as the one adopted by Gail et al. 2009) cannot obtain supersolar metallicities at the moment of the formation of the Solar System. This is an intrinsic property of one-dimensional GCE models, in which the mean metallicity is expected to grow with time. In contrast, we found with our chemo-dynamical model that metal-rich stars $\left(Z>Z_{\odot}\right)$ may migrate from the inner galaxy to the galactocentric position where the Sun formed. This automatically reduces the relative contribution from low-metallicity AGBs. Moreover, because of the long migration timescales of these stars, they also affect the mass distribution of SiC parent stars. Low-mass (1.5-2.0 $\left.M_{\odot}\right)$ metal-rich stars are favored because they evolve on long evolutionary timescales (and thus they have time to migrate). Our mass distribution is weighted toward lower initial stellar masses also because of a different TDU efficiency. In their synthetic approach, Gail et al. (2009) adopted the TDU efficiencies of AGB models by Karakas et al. (2002). These models are characterized by a higher TDU efficiency in the $3-4 M_{\odot}$ mass range with respect to our models (for a comparison, see Cristallo et al. 2011 ), resulting in the higher weight of $3-4 M_{\odot}$ in the final SiC parent AGB star distribution with respect to our results.

Recently, Lugaro et al. (2020; see also Lugaro et al. 2018) proposed that presolar $\mathrm{SiC}$ grains originated in more massive $\left(M \sim 4 M_{\odot}\right)$ and more metal-rich $\left(Z \sim 2 \times Z_{\odot}\right)$ AGB stars. It is noteworthy that the grains discussed in these papers are unusually large $(>1 \mu \mathrm{m})$ and correspond to a very minor fraction of presolar $\mathrm{SiC}$, in contrast to the much smaller grains discussed here, which are the dominant population of AGB grains. Thus, the discrepancy might be resolved if the grain sizes were metallicity dependent, that is, decreasing grain size with decreasing metallicity. This hypothesized size-metallicity correlation appears to be supported by the size-dependent $\mathrm{Sr}$ and $\mathrm{Ba}$ isotopic anomalies observed in bulk meteoritic $\mathrm{SiC}$ acid residues (Ott \& Begemann 1990; Podosek et al. 2004). In this scenario, the small population of large AGB grains is well explained by the rarity of supersolar metallicity AGB stars present in the solar vicinity at $4.57 \mathrm{Gyr}$ ago, as predicted by the MCM13 model. In comparison, $\sim 2 M_{\odot}, \sim Z_{\odot}$ AGB stars were much more common, resulting in the dominant population of small grains, as shown in Figs. 3 and 4. Interestingly, Vescovi et al. (2020) recently showed that the same set of measured AGB grain data can be well explained by magnetic FRUITY model predictions for $2 M_{\odot}$ AGB stars with close-to-solar metallicities, thus supporting our result.

Finally, we would also like to clarify the explanation to the recent proposal by Ek et al. (2019) that the Solar System $s$-process component was produced with a higher efficiency than the $s$-process component recorded in bulk AGB grains. First of all, we note that this proposal in fact is a natural consequence of GCE. During GCE, gas, the dominant form of ISM material from which the Solar System formed, preserved all its memory of $s$ process production over the previous $\sim 6.5 \mathrm{Gyr}$, while the incorporated AGB grains mostly recorded the $s$-production from the last 300 Myr prior to the Solar System formation (Heck et al. 2020). Because the efficiency of the $s$-process increases with decreasing metallicity and in turn decreases over time due to the AMR, the Solar System thus naturally contains a more efficient $s$-process component than its incorporated AGB grains. 

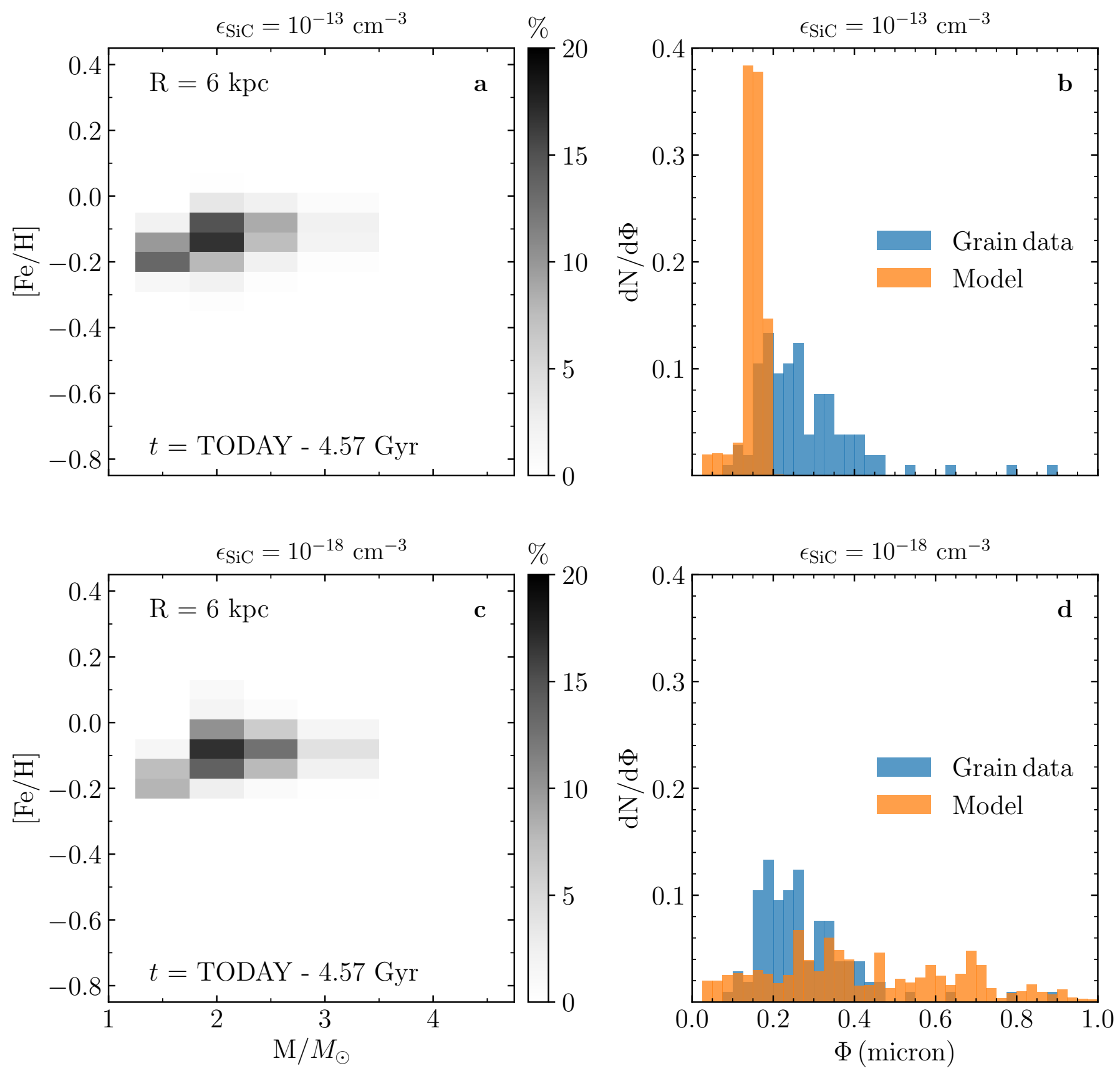

Fig. 4. Same as Fig. 3, but assuming $\epsilon_{\mathrm{SiC}}=10^{-13} \mathrm{~cm}^{-3}$ (panels $a$ and $b$ ) and $\epsilon_{\mathrm{SiC}}=10^{-18} \mathrm{~cm}^{-3}$ (panels $c$ and $d$ ). See text for details.

However, this natural difference between the Solar System and its incorporated AGB grains may not be the explanation of the small $\mathrm{Pd}$ isotopic anomalies with respect to $\mathrm{Mo}$ and $\mathrm{Ru}$ observed by Ek et al. (2019). This is because the Solar System abundances for the three elements received only $\sim 35 \%$ to $\sim 50 \%$ contributions from the $s$-process (Prantzos et al. 2020), the missing part coming from the $r$-process. The latter may have enhanced their Solar System abundances with different efficiencies, as is expected in astrophysical $r$-process sites with varying electron abundances (e.g., production of higher-thansolar $\mathrm{Mo} / \mathrm{Pd}$ and $\mathrm{Ru} / \mathrm{Pd}$ by the $r$-process with $Y_{e}$ of $0.25-0.30$; see, e.g., Lippuner \& Roberts 2015). The smaller-than-expected $\mathrm{Pd}$ isotopic anomalies observed by Ek et al. (2019) therefore do not necessarily suggest that AGB grains dominantly came from supersolar metallicity AGB stars and is irrelevant when the absolute metallicities of the parent stars of AGB grains are discussed.

\section{Conclusions and future plans}

We determined the mass and metallicity distribution of presolar $\mathrm{SiC}$ parent stars by integrating a numerical Milky Way chemodynamical model with dust yields from FRUITY models. Our models predict that the $\mathrm{SiC}$ production at the epoch of the Solar System formation is dominated by contributions from AGB stars with $M \sim 2 M_{\odot}$ and $Z \sim Z_{\odot}$, which are thus likely the parent stars of most presolar $\mathrm{SiC}$ grains identified in extraterrestrial materials.

We plan to investigate the proposed metallicity-size correlation (based on size-dependent isotopic anomalies observed in 
bulk $\mathrm{SiC}$ acid residues) by implementing our treatment of magnetic buoyancy (Vescovi et al. 2020) in all FRUITY stellar models. As shown by Vescovi et al. (2020), this treatment is needed for FRUITY models to reproduce the $s$-process isotopic signatures of AGB grains. By adopting magnetic FRUITY stellar models in the framework of the analysis presented in this paper, we will be able to provide further insights into the suggested metallicity-size correlation.

Finally, it is noteworthy that there still exist a number of unknowns in AGB modeling, such as the efficiency of TDUs, the mass-loss law, and the origin of mixing processes at work in stellar interiors. Dust nucleation (theory), describing the transition from gas-phase molecules to dust grains through molecular clusters, appears even more uncertain (Gobrecht et al. 2017). Unlike their bulk analog $(\beta-\mathrm{SiC})$, the smallest $\mathrm{SiC}$ clusters are characterized by atomic segregation of silicon and carbon atoms. It is still a matter of debate at which cluster size $\mathrm{SiC}$ favors structures with alternating $\mathrm{Si}-\mathrm{C}$ atomic ordering because the $\mathrm{SiC}$ cluster energies strongly depend on the employed level of theory (density functional). For this reason, further theoretical investigation is required, as well as a corresponding thorough experimental verification.

Acknowledgements. We thank the anonymous referee for a quick, detailed and helpful report, which largely improved the quality of our paper. A.N. acknowledges the support of the Centre National d'Etudes Spatiale (CNES) through a post-doctoral fellowship. G.C. acknowledges financial support from the EU COST Action CA16117 (ChETEC). N.L. acknowledges financial support from NASA (80NSSC20K0387 to N.L.). D.G. acknowledges support from the ERC consolidator grant 646758 AEROSOL.

\section{References}

Amari, S., Lewis, R. S., \& Anders, E. 1994, Geochim. Cosmochim. Acta, 58, 459

Anders, F., Chiappini, C., Santiago, B. X., et al. 2018, A\&A, 619, A125

Busso, M., Gallino, R., \& Wasserburg, G. J. 1999, ARA\&A, 37, 239

Cherchneff, I., Barker, J. R., \& Tielens, A. e. G. G. M. 1992, ApJ, 401, 269

Clayton, D. D. 1997, ApJ, 484, L67

Cowan, J. J., Sneden, C., Lawler, J. E., et al. 2019, Rev. Mod. Phys., submitted [arXiv:1901.01410]

Cristallo, S., Straniero, O., Gallino, R., et al. 2009, ApJ, 696, 797

Cristallo, S., Piersanti, L., Straniero, O., et al. 2011, ApJS, 197, 17

Cristallo, S., Straniero, O., Piersanti, L., \& Gobrecht, D. 2015, ApJS, 219, 40

Daulton, T. L., Bernatowicz, T. J., Lewis, R. S., et al. 2002, Science, 296, 1852

Davidson, J., Busemann, H., Nittler, L. R., et al. 2014, Geochim. Cosmochim. Acta, 139, 248

Delgado Mena, E., Tsantaki, M., Adibekyan, V. Z., et al. 2017, A\&A, 606, A94 Ek, M., Hunt, A. C., Lugaro, M., \& Schönbächler, M. 2019, Nat. Astron., 4, 273 Ferrarotti, A. S., \& Gail, H. P. 2006, A\&A, 447, 553

Floss, C., \& Stadermann, F. 2009, Geochim. Cosmochim. Acta Suppl., 73, 385
Frenklach, M., \& Feigelson, E. D. 1989, ApJ, 341, 372

Gail, H. P., Zhukovska, S. V., Hoppe, P., \& Trieloff, M. 2009, ApJ, 698, 1136 Gobrecht, D., Cristallo, S., Piersanti, L., \& Bromley, S. T. 2017, ApJ, 840, 117 Haenecour, P., Floss, C., Zega, T. J., et al. 2018, Geochim. Cosmochim. Acta, 221,379

Hanner, M. 1988, Grain optical properties (Infrared Observations of Comets Halley and Wilson and Properties of the Grains)

Heck, P. R., Greer, J., Kööp, L., et al. 2020, Proc. Nat. Acad. Sci., 117, 1884

Hoppe, P., Leitner, J., Gröner, E., et al. 2010, ApJ, 719, 1370

Hoppe, P., Stancliffe, R. J., Pignatari, M., \& Amari, S. 2019, ApJ, 887, 8

Karakas, A. I., Lattanzio, J. C., \& Pols, O. R. 2002, PASA, 19, 515

Lewis, K. M., Lugaro, M., Gibson, B. K., \& Pilkington, K. 2013, ApJ, 768, L19

Lippuner, J., \& Roberts, L. F. 2015, ApJ, 815, 82

Liu, N., Savina, M. R., Davis, A. M., et al. 2014, ApJ, 786, 66

Liu, N., Savina, M. R., Gallino, R., et al. 2015, ApJ, 803, 12

Liu, N., Nittler, L. R., Pignatari, M., O’D. Alexand er, C. M., \& Wang, J. 2017a, ApJ, 842, L1

Liu, N., Steele, A., Nittler, L. R., et al. 2017b, Meteorit. Planet. Sci., 52, 2550

Liu, N., Gallino, R., Cristallo, S., et al. 2018, ApJ, 865, 112

Liu, N., Stephan, T., Cristallo, S., et al. 2019, ApJ, 881, 28

Lucy, L. B. 1976, ApJ, 205, 482

Lugaro, M., Davis, A. M., Gallino, R., et al. 2003, ApJ, 593, 486

Lugaro, M., Karakas, A. I., Pető, M., \& Plachy, E. 2018, Geochim. Cosmochim. Acta, 221, 6

Lugaro, M., Cseh, B., Világos, B., et al. 2020, ApJ, 898, 96

Martig, M., Bournaud, F., Croton, D. J., Dekel, A., \& Teyssier, R. 2012, ApJ, 756,26

Minchev, I. 2016, Astron. Nachr., 337, 703

Minchev, I., Chiappini, C., \& Martig, M. 2013, A\&A, 558, A9

Minchev, I., Matijevic, G., Hogg, D. W., et al. 2019, MNRAS, 487, 3946

Nanni, A. 2019, MNRAS, 482, 4726

Nanni, A., Bressan, A., Marigo, P., \& Girardi, L. 2013, MNRAS, 434, 2390

Nanni, A., Marigo, P., Groenewegen, M. A. T., et al. 2016, MNRAS, 462, 1215

Nguyen, A. N., Nittler, L. R., Stadermann, F. J., Stroud, R. M., \& Alexander, C. M. O. 2010, ApJ, 719, 166

Nittler, L. R., Alexander, C. M. O., Davidson, J., et al. 2018, Geochim. Cosmochim. Acta, 226, 107

Ott, U., \& Begemann, F. 1990, ApJ, 353, L57

Piersanti, L., Cristallo, S., \& Straniero, O. 2013, ApJ, 774, 98

Pitman, K. M., Hofmeister, A. M., Corman, A. B., \& Speck, A. K. 2008, A\&A, 483, 661

Podosek, F. A., Prombo, C. A., Amari, S., \& Lewis, R. S. 2004, ApJ, 605, 960

Prantzos, N., Abia, C., Cristallo, S., Limongi, M., \& Chieffi, A. 2020, MNRAS, 491, 1832

Salpeter, E. E. 1955, ApJ, 121, 161

Savina, M. R., Pellin, M. J., Tripa, C. E., et al. 2003, Geochim. Cosmochim. Acta, 67, 3215

Stephan, T., Trappitsch, R., Davis, A. M., et al. 2016, Int. J. Mass Spectrom., 407, 1

Straniero, O., Gallino, R., \& Cristallo, S. 2006, Nucl. Phys. A, 777, 311

Ventura, P., di Criscienzo, M., Schneider, R., et al. 2012, MNRAS, 424, 2345

Vescovi, D., Cristallo, S., Busso, M., \& Liu, N. 2020, ApJ, 897, L25

Wielen, R., Fuchs, B., \& Dettbarn, C. 1996, A\&A, 314, 438

Xu, Y., Zinner, E., Gallino, R., et al. 2015, ApJ, 799, 156

Zinner, E. 2014, in Presolar Grains, ed. A. M. Davis, 1, 181

Zinner, E., Amari, S., Guinness, R., et al. 2007, Geochim. Cosmochim. Acta, 71, 4786 